\title{
Cone $A_{b}$-metric space and some coupled fixed point theo- rems
}

\author{
K. Anthony Singh ${ }^{a, *}$, M. R. Singh ${ }^{b}$, M. Bina Devi ${ }^{a}$, Th. Chhatrajit Singh ${ }^{c}$ \\ ${ }^{a}$ Department of Mathematics, D. M. College of Science, Dhanamanjuri University, Imphal (Manipur), India. \\ ${ }^{b}$ Department of Mathematics, Manipur University, Canchipur (Manipur), India. \\ ${ }^{c}$ Department of Mathematics, Manipur Technical University, Takyelpat (Manipur)-795004, India.
}

\begin{abstract}
In this paper, we extend the definition of coupled fixed point to mappings on cone $A_{b}$-metric space and prove some coupled fixed point theorems. Our results extend the coupled fixed point results of Singh and Singh [K. A. Singh, M. R. Singh, J. Math. Comput. Sci., 10 (2020), 891-905] to cone $A_{b}$-metric space. An example is also given to illustrate the validity of our result.
\end{abstract}

Keywords: Coupled fixed point, cone metric space, cone $A_{b}$-metric space.

2020 MSC: $47 \mathrm{H} 10,54 \mathrm{H} 25$.

(C)2022 All rights reserved.

\section{Introduction}

The classical Banach contraction principle which forms the foundation of metric fixed point theory has been studied and generalized in various directions by many authors. As a generalization of metric space, Huang and Zhang [5] introduced the concept of cone metric space by replacing the set of real numbers by a general Banach space $E$ which is partially ordered with respect to a cone $P \subset E$.

On the other hand, Sedghi et al. [9] generalized metric space to S-metric space and Bakhtin [2] generalized it to b-metric space. Nizar and Nabil [13] introduced the concept of $S_{b}$-metric space. The concepts of $S$-metric space and $S_{b}$-metric space are further extended to A-metric space and $A_{b}$-metric space respectively by Abbas et al. [1] and Ughade et al. [14]. Dhamodharan and Krishnakumar [3] also again extended S-metric space to cone S-metric space. Singh and Singh [10] then generalized cone $S$-metric space to cone $S_{b}$-metric space and prove some fixed point theorems. Further, Singh et al. [12] generalized cone $S_{b}$-metric space to cone $A_{b}$-metric space and proved some fixed point theorems. In the meantime, Bhaskar and Lakshmikantham [4] introduced the concept of coupled fixed point of a mapping $\mathrm{F}: \mathrm{X} \times \mathrm{X} \rightarrow \mathrm{X}$. Lakshmikantham and Ciric [6] investigated some more coupled fixed point theorems in partially ordered sets. Then, Sabetghadam et al. [8] considered the corresponding definition of coupled

\footnotetext{
${ }^{*}$ Corresponding author

Email address: anthonykumam@manipuruniv.ac.in (K. Anthony Singh)

doi: $10.22436 /$ jmcs.024.03.06
}

Received: 2020-04-10 Revised: 2021-01-28 Accepted: 2021-01-29 
fixed point for mappings on cone metric spaces and proved some coupled fixed point theorems. Singh and Singh [11] further extended these results of Sabetghadam et al. [8] to cone $S_{b}$-metric space.

The aim of this paper is to further extend the definition of coupled fixed point to mappings on cone $A_{b}$-metric space and prove some coupled fixed point theorems. Our results extend the coupled fixed point results of Singh and Singh [11] to cone $A_{b}$-metric space. We also give an example to illustrate the validity of our result.

\section{Preliminaries}

Following definitions, properties will be needed in the sequel.

Definition 2.1 ([5]). Let $E$ be a Banach space. A subset $P$ of $E$ is called a cone if and only if

1. $\mathrm{P}$ is closed, nonempty and $\mathrm{P} \neq\{0\}$;

2. $a x+b y \in P$ for all $x, y \in P$ and nonnegative real numbers $a, b$;

3. $P \cap(-P)=\{0\}$.

Given a cone $P \subset E$, we define a partial ordering $\leqslant$ in $E$ with respect to $P$ by $x \leqslant y$ if and only if $y-x \in P$. We will write $x<y$ to indicate that $x \leqslant y$ but $x \neq y$, while $x \ll y$ will stand for $y-x \in$ int.P, where int.P denotes the interior of $P$. The cone $P$ is called normal if there is a number $K>0$ such that $0 \leqslant x \leqslant y$ implies $\|x\| \leqslant K\|y\|$ for all $x, y \in E$. The least positive number satisfying the above is called the normal constant of $P$.

The cone $P$ is called regular if every increasing sequence which is bounded from above is convergent. That is, if $\left\{x_{n}\right\}$ is a sequence such that $x_{1} \leqslant x_{2} \leqslant \cdots \leqslant x_{n} \leqslant \cdots \leqslant y$ for some $y \in E$, then there is $x \in E$ such that $\left\|x_{n}-x\right\| \rightarrow 0$ as $n \rightarrow \infty$. Equivalently, the cone $P$ is regular if and only if every decreasing sequence which is bounded from below is convergent. It is well known that a regular cone is a normal cone.

Definition 2.2 ([11]). Let $X$ be a non-empty set. Suppose the mapping $d: X \times X \rightarrow E$ satisfies

1. $d(x, y) \geqslant 0$, and $d(x, y)=0$ if and only if $x=y, \forall x, y \in X$;

2. $d(x, y)=d(y, x), \forall x, y \in X$;

3. $d(x, y) \leqslant d(x, z)+d(z, y), \quad \forall x, y, z \in X$.

Then $(X, d)$ is called a cone metric space or simply CMS.

Example 2.3 ([5]). Let $E=\mathbb{R}^{2}, P=\{(x, y): x, y \geqslant 0\}, X=\mathbb{R}$ and $d: X \times X \rightarrow E$ such that

$$
d(x, y)=(|x-y|, \alpha|x-y|),
$$

where $\alpha \geqslant 0$ is a constant. Then $(X, d)$ is a cone metric space.

Definition 2.4 ([7]). Let $X$ be a non-empty set and let $b \geqslant 1$ be a given real number. A function $A: X^{n} \rightarrow[0, \infty)$ is called an $A_{b}$-metric on $X$ if for all $x_{i}, a \in X, i=1,2,3, \cdots, n$, the following conditions are satisfied

1. $A\left(x_{1}, x_{2}, \cdots, x_{n-1}, x_{n}\right) \geqslant 0$;

2. $A\left(x_{1}, x_{2}, \cdots, x_{n-1}, x_{n}\right)=0$ if and only if $x_{1}=x_{2}=\cdots=x_{n-1}=x_{n}$;

3.

$$
\begin{aligned}
A\left(x_{1}, x_{2}, \cdots, x_{n-1}, x_{n}\right) \leqslant & b\left[A\left(x_{1}, x_{1}, \cdots,\left(x_{1}\right)_{n-1}, a\right)+A\left(x_{2}, x_{2}, \cdots,\left(x_{2}\right)_{n-1}, a\right)+\cdots\right. \\
& \left.+A\left(x_{n-1}, x_{n-1}, \cdots,\left(x_{n-1}\right)_{n-1}, a\right)+A\left(x_{n}, x_{n}, \cdots,\left(x_{n}\right)_{n-1}, a\right)\right] .
\end{aligned}
$$


The pair $(X, A)$ is called an $A_{b}$-metric space.

Example $2.5([7])$. Let $X=[1, \infty)$. Define $A_{b}: X^{n} \rightarrow[0, \infty)$ by

$$
A_{b}\left(x_{1}, x_{2}, \cdots, x_{n}\right)=\sum_{i=1}^{n} \sum_{i<j}\left|x_{i}-x_{j}\right|^{2},
$$

for all $x_{i} \in X, i=1,2,3, \cdots n$. Then $\left(X, A_{b}\right)$ is an $A_{b}$-metric space with $b=2>1$.

Definition 2.6 ([12]). Suppose that $E$ is a real Banach space, $P$ is a cone in $E$ with int.P $\neq \phi$ and $\leqslant$ is partial ordering in $E$ with respect to $P$. Let $X$ be a non-empty set, and let the function $A: X^{n} \rightarrow E$ satisfy the following conditions

1. $A\left(x_{1}, x_{2}, \cdots, x_{n-1}, x_{n}\right) \geqslant 0$;

2. $A\left(x_{1}, x_{2}, \cdots, x_{n-1}, x_{n}\right)=0$ if and only if $x_{1}=x_{2}=\cdots=x_{n-1}=x_{n}$;

3.

$$
\begin{aligned}
A\left(x_{1}, x_{2}, \cdots, x_{n-1}, x_{n}\right) \leqslant & b\left[A\left(x_{1}, x_{1}, \cdots,\left(x_{1}\right)_{n-1}, a\right)+A\left(x_{2}, x_{2}, \cdots,\left(x_{2}\right)_{n-1}, a\right)+\cdots\right. \\
& +A\left(x_{n-1}, x_{n-1}, \cdots,\left(x_{n-1}\right)_{n-1}, a\right) \\
& \left.+A\left(x_{n}, x_{n}, \cdots,\left(x_{n}\right)_{n-1}, a\right)\right], \quad \forall x_{i}, a \in X, i=1,2,3, \cdots, n,
\end{aligned}
$$

where $b \geqslant 1$ is a constant. Then, the function $A$ is called a cone $A_{b}$-metric on $X$ and the pair $(X, A)$ is called a cone $A_{b}$-metric space.

We note that cone $A_{b}$-metric spaces are generalizations of cone $S_{b}$-metric spaces since every cone $S_{b}$-metric is a cone $A_{b}$-metric with $n=3$.

Example 2.7 ([12]). Let $E=\mathbb{R}^{2}$, the Euclidean plane, and $P=\{(x, y) \in E: x, y \geqslant 0\}$, a normal cone in E. Let $X=\mathbb{R}$ and $A: X^{n} \rightarrow E$ be such that $A\left(x_{1}, x_{2}, \cdots, x_{n}\right)=A_{*}\left(x_{1}, x_{2}, \cdots, x_{n}\right)(\alpha, \beta)$, where $\alpha, \beta>0$ are constants and $A_{*}$ is an $A_{b}$-metric on $X$. Then $A$ is a cone $A_{b}$-metric on $X$. In particular, we have the function

$$
A_{*}\left(x_{1}, x_{2}, \cdots, x_{n}\right)=\sum_{i=1}^{n} \sum_{i<j}\left|x_{i}-x_{j}\right|^{2}, \quad x_{i} \in X, i=1,2,3, \cdots, n,
$$

is an $A_{b}$-metric on $X$ with $b=2$. Therefore, the function

$$
A\left(x_{1}, x_{2}, \cdots, x_{n}\right)=\left(\sum_{i=1}^{n} \sum_{i<j}\left|x_{i}-x_{j}\right|^{2}, \frac{1}{4} \sum_{i=1}^{n} \sum_{i<j}\left|x_{i}-x_{j}\right|^{2}\right),
$$

is a cone $A_{b}$-metric on $X$ with $b=2$.

Definition $2.8([12])$. Let $(X, A)$ be a cone $A_{b}$-metric space.

1. A sequence $\left\{x_{n}\right\}$ in $X$ is said to converge to $x$ if for each $c \in E, 0 \ll c$ there exists $n_{0} \in \mathbb{N}$ such that for all $n \geqslant n_{0}, A\left(x_{n}, x_{n}, \cdots, x_{n}, x\right) \ll c$. We denote this by $\lim _{n \rightarrow \infty} x_{n}=x$ or $x_{n} \rightarrow x$ as $n \rightarrow \infty$.

2. A sequence $\left\{x_{n}\right\}$ in $X$ is called a Cauchy sequence if for each $c \in E, 0 \ll c$ there exists $n_{0} \in \mathbb{N}$ such that for all $n, m \geqslant n_{0}, A\left(x_{n}, x_{n}, \cdots, x_{n}, x_{m}\right) \ll c$.

3. The cone $A_{b}$-metric space $(X, A)$ is called complete if every Cauchy sequence is convergent.

Lemma 2.9 ([12]). Let $(X, A)$ be a cone $A_{b}$-metric space. Then, for all $x, y, a \in X$,

(i) $A(x, x, \cdots, x, y) \leqslant b A(y, y, \cdots, y, x)$; 
(ii) $A(x, x, \cdots, x, y) \leqslant(n-1) b A(x, x, \cdots, x, a)+b A(y, y, \cdots, y, a)$.

Lemma 2.10 ([12]). Let $(X, A)$ be a cone $A_{b}$-metric space, $\mathrm{P}$ be a normal cone with normal constant $\mathrm{K}$. Then a sequence $\left\{x_{n}\right\}$ in $X$ converges to $x$ if and only if $A\left(x_{n}, x_{n}, \cdots, x_{n}, x\right) \rightarrow 0$ as $n \rightarrow \infty$.

Lemma 2.11 ([12]). Let $(X, A)$ be a cone $A_{b}$-metric space, $\mathrm{P}$ be a normal cone with normal constant $\mathrm{K}$. Let $\left\{x_{n}\right\}$ be a sequence in $X$. If $\left\{x_{n}\right\}$ converges to $w_{1}$ and $\left\{x_{n}\right\}$ converges to $w_{2}$, then $w_{1}=w_{2}$. That is, the limit of a convergent sequence is unique.

Lemma 2.12 ([12]). Let $(\mathrm{X}, \mathrm{A})$ be a cone $\mathrm{A}_{\mathrm{b}}$-metric space, $\mathrm{P}$ be a normal cone with normal constant $\mathrm{K}$. Then a sequence $\left\{x_{n}\right\}$ in $X$ is a Cauchy sequence if and only if $A\left(x_{n}, x_{n}, \cdots, x_{n}, x_{m}\right) \rightarrow 0$ as $n, m \rightarrow \infty$.

Lemma $2.13([12])$. Let $(X, A)$ be a cone $A_{b}$-metric space, $P$ be a normal cone with normal constant $\mathrm{K}$. Let $\left\{x_{n}\right\}$ be a sequence in $X$. If $\left\{x_{n}\right\}$ converges to $w$, then $\left\{x_{n}\right\}$ is a Cauchy sequence. That is, every convergent sequence is Cauchy.

\section{Main results}

We now state and prove our main results.

First we give the corresponding definition of coupled fixed point in cone $A_{b}$-metric space.

Definition 3.1. Let $(X, A)$ be a cone $A_{b}$-metric space. An element $(x, y) \in X \times X$ is said to be a coupled fixed point of $F: X \times X \rightarrow X$ if $F(x, y)=x$ and $F(y, x)=y$.

Theorem 3.2. Let $(\mathrm{X}, \mathrm{A})$ be a complete cone $\mathrm{A}_{\mathrm{b}}$-metric space and $\mathrm{P}$ be a normal cone with normal constant $\mathrm{K}$. Suppose the mapping $\mathrm{F}: \mathrm{X} \times \mathrm{X} \rightarrow \mathrm{X}$ satisfies the following contractive condition

$$
A(F(x, y), \cdots, F(x, y), F(u, v)) \leqslant k A(x, \cdots, x, u)+l A(y, \cdots, y, v),
$$

for all $\mathrm{x}, \mathrm{y}, \mathrm{u}, v \in \mathrm{X}$, where $\mathrm{k}, \mathrm{l}$ are nonnegative constants with $\mathrm{k}+\mathrm{l} \in\left[0, \frac{1}{\mathrm{~b}^{2}}\right)$. Then, $\mathrm{F}$ has a unique coupled fixed point.

Proof. Let us choose $x_{0}, y_{0} \in X$ and set $x_{1}=F\left(x_{0}, y_{0}\right), y_{1}=F\left(y_{0}, x_{0}\right), x_{2}=F\left(x_{1}, y_{1}\right), y_{2}=F\left(y_{1}, x_{1}\right), \cdots$, $x_{n+1}=F\left(x_{n}, y_{n}\right), y_{n+1}=F\left(y_{n}, x_{n}\right)$. Then using condition (3.1), we obtain

$$
\begin{aligned}
A\left(x_{n}, \cdots, x_{n}, x_{n+1}\right) & =A\left(F\left(x_{n-1}, y_{n-1}\right), \cdots, F\left(x_{n-1}, y_{n-1}\right), F\left(x_{n}, y_{n}\right)\right) \\
& \leqslant k A\left(x_{n-1}, \cdots, x_{n-1}, x_{n}\right)+l A\left(y_{n-1}, \cdots, y_{n-1}, y_{n}\right) .
\end{aligned}
$$

Also, we have

$$
\begin{aligned}
A\left(y_{n}, \cdots, y_{n}, y_{n+1}\right) & =A\left(F\left(y_{n-1}, x_{n-1}\right), \ldots, F\left(y_{n-1}, x_{n-1}\right), F\left(y_{n}, x_{n}\right)\right) \\
& \leqslant k A\left(y_{n-1}, \cdots, y_{n-1}, y_{n}\right)+l A\left(x_{n-1}, \cdots, x_{n-1}, x_{n}\right) .
\end{aligned}
$$

If we let $A_{n}=A\left(x_{n}, \cdots, x_{n}, x_{n+1}\right)+A\left(y_{n}, \cdots, y_{n}, y_{n+1}\right)$, then we have

$$
\begin{aligned}
A_{n} & =A\left(x_{n}, \cdots, x_{n}, x_{n+1}\right)+A\left(y_{n}, \cdots, y_{n}, y_{n+1}\right) \\
& \leqslant(k+l)\left(A\left(x_{n-1}, \cdots, x_{n-1}, x_{n}\right)+A\left(y_{n-1}, \cdots, y_{n-1}, y_{n}\right)\right) \\
& =(k+l) A_{n-1} .
\end{aligned}
$$

Thus if we take $\alpha=k+l<1$, then for each $n \in \mathbb{N}$, we have

$$
0 \leqslant A_{n} \leqslant \alpha A_{n-1} \leqslant \alpha^{2} A_{n-2} \leqslant \cdots \leqslant \alpha^{n} A_{0} .
$$

If $A_{0}=0,\left(x_{0}, y_{0}\right)$ is a coupled fixed point of $F$. Let us therefore suppose that $A_{0}>0$. Then for $m>n$, we have

$$
A\left(x_{n}, \cdots, x_{n}, x_{m}\right) \leqslant b\left[(n-1) A\left(x_{n}, \cdots, x_{n}, x_{n+1}\right)+A\left(x_{m}, \cdots, x_{m}, x_{n+1}\right)\right]
$$




$$
\begin{aligned}
\leqslant & (n-1) b A\left(x_{n}, \cdots, x_{n}, x_{n+1}\right)+b^{2} A\left(x_{n+1}, \cdots, x_{n+1}, x_{m}\right) \\
\leqslant & (n-1) b A\left(x_{n}, \cdots, x_{n}, x_{n+1}\right)+(n-1) b^{3} A\left(x_{n+1}, \cdots, x_{n+1}, x_{n+2}\right) \\
& +b^{4} A\left(x_{n+2}, \cdots, x_{n+2}, x_{m}\right) \\
\leqslant & (n-1) b A\left(x_{n}, \cdots, x_{n}, x_{n+1}\right)+(n-1) b^{3} A\left(x_{n+1}, \cdots, x_{n+1}, x_{n+2}\right) \\
& +(n-1) b^{5} A\left(x_{n+2}, \cdots, x_{n+2}, x_{n+3}\right)+\cdots+b^{2(m-n-1)} A\left(x_{m-1}, \cdots, x_{m-1}, x_{m}\right) \\
\leqslant & (n-1) b\left\{A\left(x_{n}, \cdots, x_{n}, x_{n+1}\right)+b^{2} A\left(x_{n+1}, \cdots, x_{n+1}, x_{n+2}\right)\right. \\
& \left.+b^{4} A\left(x_{n+2}, \cdots, x_{n+2}, x_{n+3}\right)+\cdots+b^{2(m-n-1)} A\left(x_{m-1}, \cdots, x_{m-1}, x_{m}\right)\right\} .
\end{aligned}
$$

Similarly, we have

$$
\begin{aligned}
A\left(y_{n}, \cdots, y_{n}, y_{m}\right) \leqslant & (n-1) b\left\{A\left(y_{n}, \cdots, y_{n}, y_{n+1}\right)+b^{2} A\left(y_{n+1}, \cdots, y_{n+1}, y_{n+2}\right)\right. \\
& \left.+b^{4} A\left(y_{n+2}, \cdots, y_{n+2}, y_{n+3}\right)+\cdots+b^{2(m-n-1)} A\left(y_{m-1}, \cdots, y_{m-1}, y_{m}\right)\right\} .
\end{aligned}
$$

Therefore, we have

$$
\begin{aligned}
A\left(x_{n}, \cdots, x_{n}, x_{m}\right)+A\left(y_{n}, \cdots, y_{n}, y_{m}\right) \leqslant & (n-1) b\left\{A_{n}+b^{2} A_{n+1}+b^{4} A_{n+2}+\cdots\right. \\
& \left.+b^{2(m-n-1)} A_{m-1}\right\} \\
\leqslant & (n-1) b\left\{\alpha^{n}+b^{2} \alpha^{n+1}+b^{4} \alpha^{n+2}+\cdots\right. \\
& \left.+b^{2(m-n-1)} \alpha^{m-1}\right\} A_{0} \\
= & (n-1) b \alpha^{n}\left\{1+b^{2} \alpha+b^{4} \alpha^{2}+\cdots\right. \\
& \left.+b^{2(m-n-1)} \alpha^{m-n-1}\right\} A_{0} \\
= & (n-1) b \alpha^{n}\left\{1+b^{2} \alpha+\left(b^{2} \alpha\right)^{2}+\cdots+\left(b^{2} \alpha\right)^{m-n-1}\right\} A_{0} \\
\leqslant & \frac{(n-1) b \alpha^{n}}{1-b^{2} \alpha} A_{0} \\
\Rightarrow\left\|A\left(x_{n}, \cdots, x_{n}, x_{m}\right)+A\left(y_{n}, \cdots, y_{n}, y_{m}\right)\right\| \leqslant & \frac{(n-1) b \alpha^{n} K}{1-b^{2} \alpha}\left\|A_{0}\right\| \rightarrow 0 \text { as } n, m \rightarrow \infty .
\end{aligned}
$$

Therefore, $\left\{x_{n}\right\},\left\{y_{n}\right\}$ are Cauchy sequences in $X$. Using the completeness hypothesis, there exist $x^{*}, y^{*} \in X$ such that $\lim _{n \rightarrow \infty} x_{n}=x^{*}$ and $\lim _{n \rightarrow \infty} y_{n}=y^{*}$. We show that $\left(x^{*}, y^{*}\right)$ is a coupled fixed point of $F$. Using condition 3 of Definition 2.6 and (3.1), we have

$$
\begin{aligned}
A\left(F\left(x^{*}, y^{*}\right), \cdots, F\left(x^{*}, y^{*}\right), x^{*}\right) \leqslant & (n-1) b A\left(F\left(x^{*}, y^{*}\right), \cdots, F\left(x^{*}, y^{*}\right), x_{n+1}\right) \\
& +b A\left(x^{*}, \cdots, x^{*}, x_{n+1}\right) \\
\leqslant & (n-1) b A\left(F\left(x^{*}, y^{*}\right), \cdots, F\left(x^{*}, y^{*}\right), F\left(x_{n}, y_{n}\right)\right)+b A\left(x^{*}, \ldots, x^{*}, x_{n+1}\right) \\
\leqslant & (n-1) b k A\left(x^{*}, \cdots, x^{*}, x_{n}\right)+(n-1) b l A\left(y^{*}, \cdots, y^{*}, y_{n}\right) \\
& +b A\left(x^{*}, \cdots, x^{*}, x_{n+1}\right) \\
\Rightarrow\left\|A\left(F\left(x^{*}, y^{*}\right), \cdots, F\left(x^{*}, y^{*}\right), x^{*}\right)\right\| & K\left((n-1) b k\left\|A\left(x^{*}, \cdots, x^{*}, x_{n}\right)\right\|\right. \\
& +(n-1) b l\left\|A\left(y^{*}, \cdots, y^{*}, y_{n}\right)\right\| \\
& \left.+b\left\|A\left(x^{*}, \cdots, x^{*}, x_{n+1}\right)\right\|\right] \rightarrow 0 \text { as } n \rightarrow \infty \\
\Rightarrow & \left\|A\left(F\left(x^{*}, y^{*}\right), \cdots, F\left(x^{*}, y^{*}\right), x^{*}\right)\right\|=0 \\
\Rightarrow & A\left(F\left(x^{*}, y^{*}\right), \cdots, F\left(x^{*}, y^{*}\right), x^{*}\right)=0 \\
\Rightarrow & F\left(x^{*}, y^{*}\right)=x^{*} .
\end{aligned}
$$

Similarly, we have $F\left(y^{*}, x^{*}\right)=y^{*}$. Thus, $\left(x^{*}, y^{*}\right)$ is a coupled fixed point of $F$.

Now if $\left(x^{\prime}, y^{\prime}\right)$ is another coupled fixed point of $F$, then we have

$$
A\left(x^{\prime}, \cdots, x^{\prime}, x^{*}\right)=A\left(F\left(x^{\prime}, y^{\prime}\right), \cdots, F\left(x^{\prime}, y^{\prime}\right), F\left(x^{*}, y^{*}\right)\right)
$$




$$
\leqslant k A\left(x^{\prime}, \ldots, x^{\prime}, x^{*}\right)+l A\left(y^{\prime}, \ldots, y^{\prime}, y^{*}\right),
$$

and

$$
\begin{aligned}
A\left(y^{\prime}, \cdots, y^{\prime}, y^{*}\right) & =A\left(F\left(y^{\prime}, x^{\prime}\right), \cdots, F\left(y^{\prime}, x^{\prime}\right), F\left(y^{*}, x^{*}\right)\right) \\
& \leqslant k A\left(y^{\prime}, \cdots, y^{\prime}, y^{*}\right)+l A\left(x^{\prime}, \cdots, x^{\prime}, x^{*}\right) .
\end{aligned}
$$

Therefore, we have

$$
A\left(x^{\prime}, \ldots, x^{\prime}, x^{*}\right)+A\left(y^{\prime}, \ldots, y^{\prime}, y^{*}\right) \leqslant(k+l)\left(A\left(x^{\prime}, \ldots, x^{\prime}, x^{*}\right)+A\left(y^{\prime}, \ldots, y^{\prime}, y^{*}\right)\right) .
$$

Since $k+l<1,(3.2)$ implies that $A\left(x^{\prime}, \ldots, x^{\prime}, x^{*}\right)+A\left(y^{\prime}, \ldots, y^{\prime}, y^{*}\right)=0$. This means that

$$
A\left(x^{\prime}, \cdots, x^{\prime}, x^{*}\right)=0,
$$

and $A\left(y^{\prime}, \cdots, y^{\prime}, y^{*}\right)=0$. Hence we have $\left(x^{\prime}, y^{\prime}\right)=\left(x^{*}, y^{*}\right)$. Therefore, the coupled fixed point of $F$ is unique.

Theorem 3.3. Let $(\mathrm{X}, \mathrm{A})$ be a complete cone $\mathrm{A}_{\mathrm{b}}$-metric space and $\mathrm{P}$ be a normal cone with normal constant $\mathrm{K}$. Suppose the mapping $\mathrm{F}: \mathrm{X} \times \mathrm{X} \rightarrow \mathrm{X}$ satisfies the following contractive condition

$$
A(F(x, y), \cdots, F(x, y), F(u, v)) \leqslant k A(F(x, y), \cdots, F(x, y), x)+l A(F(u, v), \cdots, F(u, v), u),
$$

for all $x, y, u, v \in X$, where $k, l$ are nonnegative constants with $k+l \in\left[0, \frac{1}{(n-1) b^{3}}\right)$. Then, $F$ has a unique coupled fixed point.

Proof. Let us choose $x_{0}, y_{0} \in X$ and set $x_{1}=F\left(x_{0}, y_{0}\right), y_{1}=F\left(y_{0}, x_{0}\right), x_{2}=F\left(x_{1}, y_{1}\right), y_{2}=F\left(y_{1}, x_{1}\right), \cdots$, $x_{n+1}=F\left(x_{n}, y_{n}\right), y_{n+1}=F\left(y_{n}, x_{n}\right)$. Using (3.3) and condition 3 of Definition 2.6, we get

$$
\begin{aligned}
A\left(x_{n}, x_{n}, x_{n+1}\right)= & A\left(F\left(x_{n-1}, y_{n-1}\right), \cdots, F\left(x_{n-1}, y_{n-1}\right), F\left(x_{n}, y_{n}\right)\right) \\
\leqslant & k A\left(F\left(x_{n-1}, y_{n-1}\right), \cdots, F\left(x_{n-1}, y_{n-1}\right), x_{n-1}\right) \\
& +\operatorname{lA}\left(F\left(x_{n}, y_{n}\right), \cdots, F\left(x_{n}, y_{n}\right), x_{n}\right) \\
\leqslant & k A\left(x_{n}, \cdots, x_{n}, x_{n-1}\right)+l A\left(x_{n+1}, \cdots, x_{n+1}, x_{n}\right) \\
\leqslant & k b A\left(x_{n-1}, \cdots, x_{n-1}, x_{n}\right)+l b A\left(x_{n}, \cdots, x_{n}, x_{n+1}\right) \\
\Rightarrow & A\left(x_{n}, \cdots, x_{n}, x_{n+1}\right) \leqslant \frac{k b}{1-l b} A\left(x_{n-1}, \cdots, x_{n-1}, x_{n}\right) \\
\Rightarrow & A\left(x_{n}, \cdots, x_{n}, x_{n+1}\right) \leqslant \beta A\left(x_{n-1}, \cdots, x_{n-1}, x_{n}\right), \text { where } \beta=\frac{k b}{1-l b}<1 .
\end{aligned}
$$

Similarly, we have

$$
A\left(y_{n}, \cdots, y_{n}, y_{n+1}\right) \leqslant \beta A\left(y_{n-1}, \cdots, y_{n-1}, y_{n}\right) .
$$

Therefore for $p>q$, we get

$$
A\left(x_{q}, \cdots, x_{q}, x_{p}\right) \leqslant \frac{(n-1) b \beta^{q}}{1-b^{2} \beta} A\left(x_{0}, \cdots, x_{0}, x_{1}\right),
$$

and

$$
\begin{gathered}
A\left(y_{q}, \cdots, y_{q}, y_{p}\right) \leqslant \frac{(n-1) b \beta^{q}}{1-b^{2} \beta} A\left(y_{0}, \cdots, y_{0}, y_{1}\right) . \\
\Rightarrow A\left(x_{q}, \cdots, x_{q}, x_{p}\right) \rightarrow \infty \text { and } A\left(y_{q}, \cdots, y_{q}, y_{p}\right) \rightarrow \infty \text { as } q, p \rightarrow \infty .
\end{gathered}
$$

Here we note that $b^{2} \beta=\frac{k b^{3}}{1-l b}<1$. 
Therefore $\left\{x_{n}\right\}$ and $\left\{y_{n}\right\}$ are Cauchy sequences in $X$. Using the completeness hypothesis, there exist $x^{*}, y^{*} \in X$ such that $\lim _{n \rightarrow \infty} x_{n}=x^{*}$ and $\lim _{n \rightarrow \infty} y_{n}=y^{*}$. We show that $\left(x^{*}, y^{*}\right)$ is a coupled fixed point of $\mathrm{F}$.

Using condition 3 of Definition 2.6 and (3.3), we have

$$
\begin{aligned}
A\left(F\left(x^{*}, y^{*}\right), \ldots, F\left(x^{*}, y^{*}\right), x^{*}\right) \leqslant & (n-1) b A\left(F\left(x^{*}, y^{*}\right), \cdots, F\left(x^{*}, y^{*}\right), x_{n+1}\right) \\
& +b A\left(x^{*}, \cdots, x^{*}, x_{n+1}\right) \\
= & (n-1) b A\left(F\left(x^{*}, y^{*}\right), \cdots, F\left(x^{*}, y^{*}\right), F\left(x_{n}, y_{n}\right)\right) \\
& +b A\left(x^{*}, \cdots, x^{*}, x_{n+1}\right) \\
\leqslant & (n-1) b k A\left(F\left(x^{*}, y^{*}\right), \cdots, F\left(x^{*}, y^{*}\right), x^{*}\right) \\
& +(n-1) b l A\left(F\left(x_{n}, y_{n}\right), \cdots, F\left(x_{n}, y_{n}\right), x_{n}\right)+b A\left(x^{*}, \ldots, x^{*}, x_{n+1}\right) \\
\Rightarrow A\left(F\left(x^{*}, y^{*}\right), \ldots, F\left(x^{*}, y^{*}\right), x^{*}\right) \leqslant & \frac{(n-1) b l}{1-(n-1) b k} A\left(F\left(x_{n}, y_{n}\right), \cdots, F\left(x_{n}, y_{n}\right), x_{n}\right) \\
& +\frac{b}{1-(n-1) b k} A\left(x^{*}, \cdots, x^{*}, x_{n+1}\right) \\
\Rightarrow A\left(F\left(x^{*}, y^{*}\right), \cdots, F\left(x^{*}, y^{*}\right), x^{*}\right) \leqslant & \frac{(n-1) b l}{1-(n-1) b k} A\left(x_{n+1}, \cdots, x_{n+1}, x_{n}\right) \\
& +\frac{b^{2}}{1-(n-1) b k} A\left(x_{n+1}, \cdots, x_{n+1}, x^{*}\right) \\
\Rightarrow\left\|A\left(F\left(x^{*}, y^{*}\right), \cdots, F\left(x^{*}, y^{*}\right), x^{*}\right)\right\| \leqslant & K\left[\frac{(n-1) b l}{1-(n-1) b k}\left\|A\left(x_{n+1}, \cdots, x_{n+1}, x_{n}\right)\right\|\right. \\
& \left.+\frac{b^{2}}{1-(n-1) b k}\left\|A\left(x_{n+1}, \cdots, x_{n+1}, x^{*}\right)\right\|\right] \rightarrow 0 \quad \text { as } n \rightarrow \infty . \\
\Rightarrow & \left\|A\left(F\left(x^{*}, y^{*}\right), \cdots, F\left(x^{*}, y^{*}\right), x^{*}\right)\right\|=0 \\
\Rightarrow & A\left(F\left(x^{*}, y^{*}\right), \cdots, F\left(x^{*}, y^{*}\right), x^{*}\right)=0 \\
\Rightarrow & F\left(x^{*}, y^{*}\right)=x^{*} .
\end{aligned}
$$

Similarly, we can get $F\left(y^{*}, x^{*}\right)=y^{*}$. Thus $\left(x^{*}, y^{*}\right)$ is a coupled fixed point of $F$.

Now if $\left(x^{\prime}, y^{\prime}\right)$ is another coupled fixed point of $F$, then we have

$$
\begin{aligned}
A\left(x^{\prime}, \ldots, x^{\prime}, x^{*}\right)= & A\left(F\left(x^{\prime}, y^{\prime}\right), \cdots, F\left(x^{\prime}, y^{\prime}\right), F\left(x^{*}, y^{*}\right)\right) \\
\leqslant & k A\left(F\left(x^{\prime}, y^{\prime}\right), \cdots, F\left(x^{\prime}, y^{\prime}\right), x^{\prime}\right) \\
& +\operatorname{lA}\left(F\left(x^{*}, y^{*}\right), \cdots, F\left(x^{*}, y^{*}\right), x^{*}\right) \\
= & 0 .
\end{aligned}
$$

Therefore, $A\left(x^{\prime}, \ldots, x^{\prime}, x^{*}\right)=0$ and so $x^{\prime}=x^{*}$. Similarly, we can get $y^{\prime}=y^{*}$. Hence we have $\left(x^{\prime}, y^{\prime}\right)=$ $\left(x^{*}, y^{*}\right)$ which shows that the coupled fixed point of $F$ is unique.

Theorem 3.4. Let $(\mathrm{X}, \mathrm{A})$ be a complete cone $\mathrm{A}_{\mathrm{b}}$-metric space and $\mathrm{P}$ be a normal cone with normal constant $\mathrm{K}$. Suppose the mapping $\mathrm{F}: \mathrm{X} \times \mathrm{X} \rightarrow \mathrm{X}$ satisfies the following contractive condition

$$
A(F(x, y), \cdots, F(x, y), F(u, v)) \leqslant k A(F(x, y), \cdots, F(x, y), u)+l A(F(u, v), \cdots, F(u, v), x),
$$

for all $x, y, u, v \in X$, where $k, l$ are nonnegative constants with $k+l \in\left[0, \frac{1}{(n-1)^{2} b^{3}}\right)$. Then, $F$ has a unique coupled fixed point.

Proof. Let us choose $x_{0}, y_{0} \in X$ and set $x_{1}=F\left(x_{0}, y_{0}\right), y_{1}=F\left(y_{0}, x_{0}\right), x_{2}=F\left(x_{1}, y_{1}\right), y_{2}=F\left(y_{1}, x_{1}\right), \cdots$, $x_{n+1}=F\left(x_{n}, y_{n}\right), y_{n+1}=F\left(y_{n}, x_{n}\right)$. Using (3.4) and Condition 3 of Definition 2.6, we get

$$
A\left(x_{n}, \cdots, x_{n}, x_{n+1}\right)=A\left(F\left(x_{n-1}, y_{n-1}\right), \cdots, F\left(x_{n-1}, y_{n-1}\right), F\left(x_{n}, y_{n}\right)\right)
$$




$$
\begin{aligned}
\leqslant & k A\left(F\left(x_{n-1}, y_{n-1}\right), \cdots, F\left(x_{n-1}, y_{n-1}\right), x_{n}\right) \\
& +\operatorname{lA}\left(F\left(x_{n}, y_{n}\right), \cdots, F\left(x_{n}, y_{n}\right), x_{n-1}\right) \\
= & k A\left(x_{n}, \cdots, x_{n}, x_{n}\right)+l A\left(x_{n+1}, \cdots, x_{n+1}, x_{n-1}\right) \\
= & l A\left(x_{n+1}, \cdots, x_{n+1}, x_{n-1}\right) \\
\leqslant & l\left((n-1) b A\left(x_{n+1}, \cdots, x_{n+1}, x_{n}\right)+b A\left(x_{n-1}, \cdots, x_{n-1}, x_{n}\right)\right) \\
\leqslant & l\left((n-1) b^{2} A\left(x_{n}, \cdots, x_{n}, x_{n+1}\right)+b A\left(x_{n-1}, \cdots, x_{n-1}, x_{n}\right)\right) \\
\Rightarrow A\left(x_{n}, \cdots, x_{n}, x_{n+1}\right) \leqslant & \frac{l b}{1-(n-1) l b^{2}} A\left(x_{n-1}, \cdots, x_{n-1}, x_{n}\right) \\
\Rightarrow A\left(x_{n}, \cdots, x_{n}, x_{n+1}\right) \leqslant & \delta A\left(x_{n-1}, \cdots, x_{n-1}, x_{n}\right) \quad \text { where } \delta=\frac{l b}{1-(n-1) l b^{2}}<1 .
\end{aligned}
$$

Similarly, we can get

$$
A\left(y_{n}, \cdots, y_{n}, y_{n+1}\right) \leqslant \delta A\left(y_{n-1}, \cdots, y_{n-1}, y_{n}\right) .
$$

Therefore for $p>q$, we get

$$
A\left(x_{q}, \cdots, x_{q}, x_{p}\right) \leqslant \frac{(n-1) b \delta^{q}}{1-b^{2} \delta} A\left(x_{0}, \cdots, x_{0}, x_{1}\right),
$$

and

$$
\begin{gathered}
A\left(y_{q}, \cdots, y_{q}, y_{p}\right) \leqslant \frac{(n-1) b \delta^{q}}{1-b^{2} \delta} A\left(y_{0}, \cdots, y_{0}, y_{1}\right) . \\
\Rightarrow A\left(x_{q}, \cdots, x_{q}, x_{p}\right) \rightarrow \infty \text { and } A\left(y_{q}, \cdots, y_{q}, y_{p}\right) \rightarrow \infty \text { as } q, p \rightarrow \infty .
\end{gathered}
$$

Here we note that $b^{2} \delta=\frac{l b^{3}}{1-(n-1) l b^{2}}<1$.

Therefore $\left\{x_{n}\right\}$ and $\left\{y_{n}\right\}$ are Cauchy sequences in $X$. Using the completeness hypothesis, there exist $x^{*}, y^{*} \in X$ such that $\lim _{n \rightarrow \infty} x_{n}=x^{*}$ and $\lim _{n \rightarrow \infty} y_{n}=y^{*}$. We show that $\left(x^{*}, y^{*}\right)$ is a coupled fixed point of $F$.

Using condition 3 of Definition 2.6 and (3.4), we have

$$
\begin{aligned}
& A\left(F\left(x^{*}, y^{*}\right), \cdots, F\left(x^{*}, y^{*}\right), x^{*}\right) \leqslant(n-1) b A\left(F\left(x^{*}, y^{*}\right), \cdots, F\left(x^{*}, y^{*}\right), x_{n+1}\right) \\
&+b A\left(x^{*}, \cdots, x^{*}, x_{n+1}\right) \\
&=(n-1) b A\left(F\left(x^{*}, y^{*}\right), \cdots, F\left(x^{*}, y^{*}\right), F\left(x_{n}, y_{n}\right)\right) \\
&+b A\left(x^{*}, \cdots, x^{*}, x_{n+1}\right) \\
& \leqslant(n-1) b k A\left(F\left(x^{*}, y^{*}\right), \cdots, F\left(x^{*}, y^{*}\right), x_{n}\right) \\
&\left.+(n-1) b l A\left(F\left(x_{n}, y_{n}\right), \cdots, F\left(x_{n}, y_{n}\right), x^{*}\right)\right)+b A\left(x^{*}, \cdots, x^{*}, x_{n+1}\right) \\
& \leqslant\left.(n-1) b k A\left(F\left(x^{*}, y^{*}\right), \cdots, F\left(x^{*}, y^{*}\right), x_{n}\right)\right) \\
&+(n-1) b l A\left(x_{n+1}, \cdots, x_{n+1}, x^{*}\right)+b^{2} A\left(x_{n+1}, \cdots, x_{n+1}, x^{*}\right) \\
& \leqslant(n-1)^{2} b^{2} k A\left(F\left(x^{*}, y^{*}\right), \cdots, F\left(x^{*}, y^{*}\right), x^{*}\right) \\
&+(n-1) b^{2} k A\left(x_{n}, \cdots, x_{n}, x^{*}\right) \\
&+\left((n-1) b l+b^{2}\right) A\left(x_{n+1}, \cdots, x_{n+1}, x^{*}\right) \\
& \Rightarrow A\left(F\left(x^{*}, y^{*}\right), \cdots, F\left(x^{*}, y^{*}\right), x^{*}\right) \leqslant \frac{(n-1) b^{2} k}{1-(n-1)^{2} b^{2} k} A\left(x_{n}, \cdots, x_{n}, x^{*}\right) \\
& \quad+\frac{(n-1) b l+b^{2}}{1-(n-1)^{2} b^{2} k} A\left(x_{n+1}, \cdots, x_{n+1}, x^{*}\right)
\end{aligned}
$$




$$
\begin{aligned}
\Rightarrow\left\|A\left(F\left(x^{*}, y^{*}\right), \cdots, F\left(x^{*}, y^{*}\right), x^{*}\right)\right\| \leqslant & K\left[\frac{(n-1) b^{2} k}{1-(n-1)^{2} b^{2} k}\left\|A\left(x_{n}, \cdots, x_{n}, x^{*}\right)\right\|\right. \\
& \left.+\frac{(n-1) b l+b^{2}}{1-(n-1)^{2} b^{2} k}\left\|A\left(x_{n+1}, \cdots, x_{n+1}, x^{*}\right)\right\|\right] \rightarrow 0 \text { as } n \rightarrow \infty \\
& \Rightarrow\left\|A\left(F\left(x^{*}, y^{*}\right), \cdots, F\left(x^{*}, y^{*}\right), x^{*}\right)\right\|=0 \\
& \Rightarrow A\left(F\left(x^{*}, y^{*}\right), \cdots, F\left(x^{*}, y^{*}\right), x^{*}\right)=0 \\
& \Rightarrow F\left(x^{*}, y^{*}\right)=x^{*} .
\end{aligned}
$$

Similarly, we can get $F\left(y^{*}, x^{*}\right)=y^{*}$. Thus $\left(x^{*}, y^{*}\right)$ is a coupled fixed point of $F$.

Now if $\left(x^{\prime}, y^{\prime}\right)$ is another coupled fixed point of $F$, then we have

$$
\begin{aligned}
A\left(x^{\prime}, \cdots, x^{\prime}, x^{*}\right)= & A\left(F\left(x^{\prime}, y^{\prime}\right), \cdots, F\left(x^{\prime}, y^{\prime}\right), F\left(x^{*}, y^{*}\right)\right) \\
\leqslant & k A\left(F\left(x^{\prime}, y^{\prime}\right), \cdots, F\left(x^{\prime}, y^{\prime}\right), x^{*}\right) \\
& +\operatorname{lA}\left(F\left(x^{*}, y^{*}\right), \cdots, F\left(x^{*}, y^{*}\right), x^{\prime}\right) \\
= & k A\left(x^{\prime}, \cdots, x^{\prime}, x^{*}\right)+l A\left(x^{*}, \cdots, x^{*}, x^{\prime}\right) \\
\leqslant & (k+l b) A\left(x^{\prime}, \cdots, x^{\prime}, x^{*}\right) .
\end{aligned}
$$

Since $k+l b<1$, the above inequality implies $A\left(x^{\prime}, \ldots, x^{\prime}, x^{*}\right)=0$ and so $x^{\prime}=x^{*}$. Similarly, we can get $y^{\prime}=y^{*}$. Hence we have $\left(x^{\prime}, y^{\prime}\right)=\left(x^{*}, y^{*}\right)$ which shows that the coupled fixed point of $F$ is unique.

When $k=l$ in Theorems 3.2, 3.3 and 3.4, we get the following corollaries.

Corollary 3.5. Let $(\mathrm{X}, \mathrm{A})$ be a complete cone $\mathrm{A}_{\mathrm{b}}$-metric space and $\mathrm{P}$ be a normal cone with normal constant $\mathrm{K}$. Suppose the mapping $\mathrm{F}: \mathrm{X} \times \mathrm{X} \rightarrow \mathrm{X}$ satisfies the following contractive condition

$$
A(F(x, y), \cdots, F(x, y), F(u, v)) \leqslant k(A(x, \cdots, x, u)+A(y, \cdots, y, v)),
$$

for all $\mathrm{x}, \mathrm{y}, \mathrm{u}, v \in \mathrm{X}$, where $\mathrm{k} \in\left[0, \frac{1}{2 \mathrm{~b}^{2}}\right)$ is a constant. Then, $\mathrm{F}$ has a unique coupled fixed point.

Corollary 3.6. Let $(\mathrm{X}, \mathrm{A})$ be a complete cone $\mathrm{A}_{\mathrm{b}}$-metric space and $\mathrm{P}$ be a normal cone with normal constant $\mathrm{K}$. Suppose the mapping $\mathrm{F}: \mathrm{X} \times \mathrm{X} \rightarrow \mathrm{X}$ satisfies the following contractive condition

$$
A(F(x, y), \cdots, F(x, y), F(u, v)) \leqslant k(A(F(x, y), \cdots, F(x, y), x)+A(F(u, v), \cdots, F(u, v), u)),
$$

for all $x, y, u, v \in X$, where $k \in\left[0, \frac{1}{2(n-1) b^{3}}\right)$ is a constant. Then, $F$ has a unique coupled fixed point.

Corollary 3.7. Let $(\mathrm{X}, \mathrm{A})$ be a complete cone $\mathrm{A}_{\mathrm{b}}$-metric space and $\mathrm{P}$ be a normal cone with normal constant $\mathrm{K}$. Suppose the mapping $\mathrm{F}: \mathrm{X} \times \mathrm{X} \rightarrow \mathrm{X}$ satisfies the following contractive condition

$$
A(F(x, y), \cdots, F(x, y), F(u, v)) \leqslant k(A(F(x, y), \cdots, F(x, y), u)+A(F(u, v), \cdots, F(u, v), x)),
$$

for all $x, y, u, v \in X$, where $k \in\left[0, \frac{1}{2(n-1)^{2} b^{3}}\right)$ is a constant. Then, $\mathrm{F}$ has a unique coupled fixed point.

Here we give an example to illustrate Corollary 3.5 .

Example 3.8. Let $E=\mathbb{R}$, the Euclidean plane, and $P=\{(x, y) \in E: x, y \geqslant 0\}$, a normal cone in $E$. Let $X=\mathbb{R}$ and $A: X^{n} \rightarrow E$ such that

$$
A\left(x_{1}, x_{2}, \cdots, x_{n}\right)=\left(\sum_{i=1}^{n-1}\left|x_{i}-x_{n}\right|, \sum_{i=1}^{n-1}\left|x_{i}-x_{n}\right|\right),
$$


$x_{i} \in X, i=1,2,3, \cdots, n$. Then $A$ is a cone $A_{b}$-metric on $X$ with $b=1$ and $(X, A)$ is a complete cone $A_{b}$-metric space. Let us consider the mapping $F: X \times X \rightarrow X$ defined by $F(x, y)=\frac{x+y}{5}$. Then we have

$$
\begin{aligned}
A(F(x, y), \cdots, F(x, y), F(u, v)) & =A\left(\frac{x+y}{5}, \cdots, \frac{x+y}{5}, \frac{u+v}{5}\right) \\
& =\left((n-1)\left|\frac{x+y}{5}-\frac{u+v}{5}\right|,(n-1)\left|\frac{x+y}{5}-\frac{u+v}{5}\right|\right) \\
& =\frac{(n-1)}{5}(|x+y-u-v|,|x+y-u-v|) \\
& \leqslant \frac{(n-1)}{5}((|x-u|,|x-u|)+(|y-v|,|y-v|)),
\end{aligned}
$$

and

$$
\begin{aligned}
A(x, \cdots, x, u)+A(y, \cdots, y, v)= & ((n-1)|x-u|,(n-1)|x-u|) \\
& +((n-1)|y-v|,(n-1)|y-v|) \\
= & (n-1)((|x-u|,|x-u|)+(|y-v|,|y-v|)) .
\end{aligned}
$$

Therefore we have

$$
A(F(x, y), \cdots, F(x, y), F(u, v)) \leqslant k(A(x, \cdots, x, u)+A(y, \cdots, y, v)),
$$

for $k=\frac{1}{5} \in\left[0, \frac{1}{2 b^{2}}\right)=\left[0, \frac{1}{2}\right)$. Thus the condition of Corollary 3.5 is satisfied and $F$ has a unique coupled fixed point $(0,0)$.

\section{References}

[1] M. Abbas, B. Ali, Y. I. Suleiman, Generalized coupled common fixed point results in partially ordered A-metric space, Fixed Point Theory Appl., 2015 (2015), 24 pages. 1

[2] I. A. Bakhtin, The contraction mapping principle in quasimetric spaces, Funct. Anal. Ulianowsk Gos. Ped. Inst., 30 (1989), 26-37. 1

[3] D. Dhamodharan, R. Krishnakumar, Cone S-Metric Space and Fixed Point Theorems of Contractive Mappings, Ann. Pure Appl. Math., 14 (2017), 237-243. 1

[4] T. Gnana Bhaskar, V. Lakshmikantham, Fixed point in partially ordered metric spaces and applications, Nonlinear Anal., 65 (2006), 1379-1393. 1

[5] L.-G. Huang, X. Zhang, Cone metric spaces and fixed point theorem of contractive mappings, J. Math. Anal. Appl., 332 (2007), 1468-1476. 1, 2.1, 2.3

[6] V. Lakshmikantham, L. Ćirić, Coupled fixed point theorems for nonlinear contractions in partially ordered metric spaces, Nonlinear Anal., 70 (2009), 4341-4349. 1

[7] N. Priyobarta, Y. Rohen, N. Mlaiki, Complex valued Sb-metric spaces, J. Math. Anal., 8 (2017), 13-24. 2.4 , 2.5

[8] F. Sabetghadam, H. P. Masiha, A. H. Sanatpour, Some coupled fixed point theorems in cone metric spaces, Fixed Point Theory Appl., 2009 (2009), 8 pages. 1

[9] S. Sedghi, N. Shobe, A. Aliouche, A generalization of fixed point in S-metric spaces, Mat. Vesnik, 64 (2012), 258-266. 1

[10] K. A. Singh, M. R. Singh, Some fixed point theorems of cone Sb-metric space, J. Indian Acad. Math., 40 (2018), $255-272$. 1

[11] K. A. Singh, M. R. Singh, Some coupled fixed point theorems in cone Sb-metric space, J. Math. Comput. Sci., 10 (2020), 891-905. 1, 2.2

[12] K. A. Singh, M. R. Singh, T. Chhatrajit Singh, Some fixed point theorems in cone Ab-metric space, Electron. J. Math. Anal. Appl., 9 (2021), 112-123. 1, 2.6, 2.7, 2.8, 2.9, 2.10, 2.11, 2.12, 2.13

[13] N. Souayah, N. Mlaiki, A fixed point theorem in Sb-metric spaces, J. Math. Computer Sci., 16 (2016), 131-139. 1

[14] M. Ughade, D. Turkoglu, S. Raj Singh, R. D. Daheriya, Some fixed point theorems in Ab-metric space, British J. Math. Comput. Sci., 19 (2016), 1-24. 1 